\title{
CONNECTIVITY PROPERTIES FOR COMPLEMENTS OF EXCEPTIONAL SETS
}

\author{
KIRSTI OJA
}

\section{Introduction}

We consider a $\mathscr{P}$-harmonic space $X$ with a countable base in the sense of Constantinescu-Cornea [3]. The following problem is studied here: Let $X$ be connected and $E$ an exceptional set of $X$. Under what circumstances is $X \backslash E$ not connected? It is known that this is never the case if $E$ is polar. But this is possible if $E$ is totally thin, as is shown by examples.

In Section 1 we derive a necessary condition for the complement of a closed totally thin set $E$ to be nonconnected, in terms of absorbent sets and the boundary of $X \backslash E$ [Theorem 1.5]. This condition is never valid in an elliptic space. For the case where the exceptional set is assumed to consist of irregular (or even unstable) boundary points of some open set, we obtain a more precise necessary condition in Section 2 [Corollary 2.9].

\section{Connectivity of the complement of a totally thin set}

Let $X$ be a Constantinescu-Cornea $\mathscr{P}$-harmonic space with a countable base. For unexplained symbols in this article we refer to [3].

Let $A \subset X$. Then

$$
b(A)=\{x \in X \mid A \text { is not thin at } x\} .
$$

Let $U$ be an open set of $X$. We denote by $U_{\text {reg }}$ (respectively $U_{i r}$ ) the set of regular (respectively irregular) points of $\partial U$. Then by [3, Theorem 6.3.3]

$$
U_{\text {reg }}=\partial U \cap b(X \backslash U), U_{i r}=\partial U \backslash b(X \backslash U) .
$$

It is known that $X \backslash E$ is connected if $X$ is connected and $E$ is a polar set of $X$ [3, Proposition 6.2.5]. In what follows we shall investigate the modified situation if $E$ is totally thin. In this case $X \backslash E$ may be nonconnected, as the following examples show.

This work was supported by the Väisälä Foundation, Helsinki. 
Example 1.1. Let $X=\mathbf{R}^{2}$ have the harmonic structure corresponding to the heat equation and

$$
E=\{(x, t) \in X \mid t=0\} .
$$

Then $X \backslash E$ is not connected and $E$ is totally thin [3, Exercise 6.3.8].

Example 1.2. Let $X$ be the space defined in [3, Exercise 3.2.13] and

$$
E=\{(0,0,0)\}
$$

Then $E$ is a polar set of the absorbent set $A$ of $X$ :

$$
A=\left\{(x, y, 0) \in X \mid 0 \leqq x^{2}+y^{2}<1\right\} .
$$

Hence $E$ is totally thin in $X$ [3, Exercise 6.3.13], and $X \backslash E$ is not connected.

Throughout this paper, we shall use the following result of Berg [1, Théorème 1]; see also [3, Exercise 7.2.11]: $A$ set which is closed and finely open is absorbent.

Lemma 1.3. Let $X$ be connected and $E$ a closed set of $X$ such that $X \backslash E=$ $U_{1} \cup U_{2}$, with $U_{1}, U_{2}$ open, nonempty, and disjoint. Let $x \in\left(U_{1}\right)_{\text {reg }}$ and $x \in\left(U_{2}\right)_{\text {reg }}$. Then $x \in(X \backslash E)_{\text {reg }}$.

Proof. Let $U^{\prime}=X \backslash E=U_{1} \cup U_{2}$ and $f \in \mathscr{K}\left(\partial U^{\prime}\right)$ be arbitrary. Then

$$
h=\left\{\begin{array}{lll}
H_{f}^{U_{1}} & \text { on } & U_{1} \\
H_{f}^{U_{2}} & \text { on } & U_{2}
\end{array}\right.
$$

is harmonic on $U^{\prime}$ and $\lim _{U^{\prime} \ni y \rightarrow x} h(y)=f(x)$. Thus $h \leqq H_{f}^{U^{\prime}}$, since $h \in \mathscr{U}_{f}^{U^{\prime}}$, and $H_{f}^{U^{\prime}} \leqq h$, since $h \in \overline{\mathscr{U}}_{f}^{U^{\prime}}$, and therefore $\lim _{U^{\prime} \ni y \rightarrow x} H_{f}^{U^{\prime}}(y)=f(x)$. The conclusion follows.

Lemma 1.4. With the notations of Lemma 1.3, let $x \in E$ and $E$ be thin at $x$. Then either $x \in b\left(U_{1}\right) \cap\left(X \backslash b\left(U_{2}\right)\right)$ or $x \in \ell\left(U_{2}\right) \cap\left(X \backslash \ell\left(U_{1}\right)\right)$.

Proof. If $x \in \partial U_{1} \cap U_{2}$, then $x \in b\left(U_{2}\right)$. If now $x \in b\left(U_{1}\right)$, then $x$ is in the fine closure of $U_{1}$. Hence $x \in \bar{U}_{1}$ and so $U_{1} \cap U_{2} \neq \emptyset$, a contradiction. Therefore we may assume $x \in \partial U_{1} \cap \partial U_{2}$.

Since $E$ is thin at $x, x$ is not a regular point for $X \backslash E$. Thus by Lemma 1.3 either $x \notin\left(U_{1}\right)_{\text {reg }}$ or $x \notin\left(U_{2}\right)_{\text {reg }}$. Let $x \notin\left(U_{1}\right)_{\text {reg }}$. Then $X \backslash U_{1}$ is thin at $x$. We must have $x \in \ell\left(U_{1}\right)$, otherwise $X=U_{1} \cup\left(X \backslash U_{1}\right)$ would be thin at $x$, by [3, Theorem 6.3.1] a contradiction. Since $U_{2} \subset X \backslash U_{1}$ and $X \backslash U_{1}$ is thin at $x$, we have $x \in X \backslash b\left(U_{2}\right)$.

By a local non-trivial absorbent set of $X$ we mean hereafter an absorbent set with respect to some open subspace of $X$, which does not reduce to a union of components of this subspace.

Theorem 1.5. Let $X$ be connected and $E$ a closed totally thin set. If $X \backslash E$ is not connected, there exists a local non-trivial absorbent set whose boundary intersects $E$ in a set containing points interior to $E$ in its relative topology. 
Proof. There exist nonempty open sets $U_{1}, U_{2}$ such that $U_{1} \cap U_{2}=\emptyset$ and $X \backslash E=U_{1} \cup U_{2}$. We can assume that $E=\partial \bar{U}_{1} \cap \partial \bar{U}_{2}$. If this is not the case, denote $E^{\prime}=E \cap \partial \bar{U}_{1}$, or, equivalently, $E^{\prime}=E \cap \partial \bar{U}_{2}$. Then $X \backslash E^{\prime}$ is not connected. If the assertion can be proved for $E^{\prime}$, it also holds for $E$, since $E^{\prime} \subset E$.

Let

$$
A_{1}=\ell\left(U_{1}\right) \cap E, \quad A_{2}=\ell\left(U_{2}\right) \cap E .
$$

Then $E=A_{1} \cup A_{2}$ and $A_{1} \cap A_{2}=\emptyset$ by Lemma 1.4. The sets $A_{1}, A_{2}$ are $G_{\delta}$-sets in the relative topology of $E$ [3, Corollary 7.2.1]. Let $\left\{G_{m}\right\}_{m \in \mathbf{N}},\left\{H_{n}\right\}_{n \in \mathbf{N}}$ be open sets in $E$ with

$$
A_{1}=\bigcap_{m \in \mathrm{N}} G_{m}, \quad A_{2}=\bigcap_{n \in \mathrm{N}} H_{n} .
$$

We shall prove that either $A_{1}$ or $A_{2}$ contains interior points. Assume the contrary. Then $A_{1}$ and $A_{2}$ both are dense on $E$. Hence $G_{m}$ and $H_{n}$ are dense open sets of $E$ for every $m, n \in \mathbf{N}$. Since $E$ is a Baire space, by local compactness,

$$
\left(\cap_{m \in \mathrm{N}} G_{m}\right) \cap\left(\cap_{n \in \mathrm{N}} H_{n}\right)=A_{1} \cap A_{2}
$$

is dense on $E$, a contradiction.

So there exists an open non-empty subset $D$ of $E$, for example, with $D \subset A_{1} \backslash A_{2}$. Then $D \subset b\left(U_{1}\right)$ and $D \cap \ell\left(U_{2}\right)=\emptyset$. Thus $D$ is contained in the fine interior of $\bar{U}_{1}$. Using the result of Berg [1, Théorème 1] we see that $\bar{U}_{1} \cap((X \backslash E) \cup D)$ is an absorbent set of $(X \backslash E) \cup D$. Since $E=\partial \bar{U}_{1} \cap \partial \bar{U}_{2}$, we have $D \subset \bar{U}_{1} \cap \overline{\left(X \backslash \bar{U}_{1}\right)}$. Thus $D$ is the boundary of this absorbent set. This is consequently non-trivial.

Corollary 1.6. Let $X$ be connected and elliptic. If $E$ is a closed totally thin set, then $X \backslash E$ is connected.

For an open subset $U$ of $X$, the set $U_{i r}$ is semi-polar [3, Corollary 7.2.2]. There is a better result that holds for closed subsets of $\partial U$ containing only points of $U_{i r}$.

Lemma 1.7. Let $U$ be an open subset of $X$ and $S$ a closed subset of $\partial U$ with $S \subset U_{i r}$. Then $S$ is totally thin.

Proof. Since $X \backslash U$ is thin at every point of $U_{i r}, S$ is thin at every point of $U_{i r}$. Also, $S$ is thin at every point of $U$ and at every point of $X \backslash \bar{U}$. Finally, let $x \in U_{\text {reg }}$. Since $S$ is closed in $\partial U, x$ has a neighbourhood which does not intersect $S$. Hence $S$ is thin at $x$. Thus $S$ is totally thin.

Corollary 1.8. Let $U$ be an open set of $X$ and $B$ an open set of $\partial \bar{U}$ with $B \subset U_{i r}$. If there exists no nontrivial local absorbent set whose boundary intersects $B$, then $B=\emptyset$.

Proof. Let $B \neq \emptyset$ and $Y$ an open, connected set of $X$ such that $Y \cap \partial \bar{U}$ is an open set of $\partial \bar{U}$ and $B \supset Y \cap \partial \bar{U}$. Thus $Y \cap \partial \bar{U} \subset U_{i r}, Y \cap \partial \bar{U}$ is closed in $Y$,

$$
Y \backslash \partial \bar{U}=(Y \backslash \bar{U}) \cup(U \cap Y),
$$

and by the assumption both sets on the right are nonempty. This implies that $Y \backslash \partial \bar{U}$ is not connected. But $Y \cap \partial \bar{U}$ is totally thin by Lemma 1.7. By Theorem 1.5 we obtain $B=\emptyset$. 


\section{On unstable and irregular points}

In this section we shall investigate the role of irregular points more closely.

Let $U$ be an open relatively compact set of $X$. Any point of $\partial U$ which lies in the fine closure of $X \backslash \bar{U}$ is a stable point of $U$ [2, p. 102], [4, Theorem 4.3]. The set of stable points of $U$ is denoted by $U_{s}$. Then by [2, p. 102]

$$
U_{s}=\partial U \cap \ell(X \backslash \bar{U}) \text {. }
$$

Obviously $U_{s} \subset U_{\text {reg. }}$. Let $S(U)$ denote the set of real continuous functions on the closure of an open set $U$ which are superharmonic on $U$ and $\mathrm{Ch}_{S(U)} \bar{U}$ denote the Choquet boundary of $S(U)[2$, p. 87]. Then

$$
U_{s} \subset \mathrm{Ch}_{S(U)} \bar{U} \subset U_{r e g} \text {. }
$$

The first inequality was established in [2, Corollary 3.5]. The second one follows from the definitions in [2].

Let $S$ be a subset of $\partial \bar{U}$. We say that the absorbent condition holds for $S$ if every $x \in \partial \bar{U} \backslash \bar{S}$ has an open neighbourhood $V$ with $\partial \bar{U} \cap \bar{V} \subset \partial U \backslash \bar{S}$ such that $\bar{U} \cap V$ is a non-trivial absorbent set of $V$.

In a global form, this is equivalent to the statement " $\bar{U} \backslash \bar{S}$ is a non-trivial absorbent set of $X \backslash \bar{S}$ ". This follows from the fact that if the condition holds, then, by the sheaf property of hyperharmonic functions, the function which is 0 on $\bar{U} \backslash \bar{S}$ and $\infty$ on $(X \backslash \bar{S}) \backslash \bar{U}$ is hyperharmonic on $X$. The converse is obvious.

The set $S$ may be empty. In this case the condition signifies that $U$ is a non-trivial absorbent set of $X$.

Proposition 2.1. Either the set $U_{s}$ is dense on $\partial \bar{U}$, or the absorbent condition holds for $U_{s}$.

Proof. Assume that $U_{s}$ is not dense on $\partial \bar{U}$. Let $V$ be an open set with $\partial \bar{U} \cap V$ containing only nonstable points of $U$ and $x \in \partial \bar{U} \cap V$. Then $x$ is not in the fine closure of $X \backslash \bar{U}$. Since $x \in \bar{U}, x$ must be a fine interior point of $\bar{U}$. This holds for every $x \in \partial \bar{U} \cap V$. Thus $V \cap \bar{U}$ is finely open in $V$ and hence an absorbent set in $V$. Since $\partial(V \cap \bar{U}) \neq \emptyset$, it is non-trivial.

Corollary 2.2. Either the set $\mathrm{Ch}_{S(U)} \bar{U}$ (respectively $U_{\text {reg}}$ ) is dense on $\partial \bar{U}$, or the absorbent condition holds for $\mathrm{Ch}_{S(U)} \bar{U}$ (respectively $U_{\text {reg }}$ ).

Proof. Obvious by (2.1).

Corollary 2.3. If $X$ is elliptic, the sets $U_{s}, \mathrm{Ch}_{S(U)} \bar{U}$ and $U_{\text {reg }}$ are dense on $\partial \bar{U}$.

Remark 2.4. Corollary 2.3 generalizes a result for $U_{\text {reg }}$ in [5, Théorème 8.2]; see also [3, Exercise 3.1.16]. The corresponding result for $U_{s}$ was proved in another way in [7, Corollary 3]. 
Proposition 2.5. Let $B$ be an open subset of $\partial \bar{U}$. Then $B \subset \partial \bar{U} \backslash U_{s}$ if and only if the absorbent condition holds for $\partial \bar{U} \backslash B$.

Proof. By Proposition 2.1 we only have to prove that if $x \in B$, with $V$ open such that $x \in V \cap \partial \bar{U} \subset B$ and $V \cap \bar{U}$ an absorbent set of $V$, then $x \notin U_{s}$. Since $V \cap \bar{U}$ is finely open, $x$ is not in the fine closure of $X \backslash \bar{U}$. The conclusion follows.

An irregular point $x \in \partial U$ is semi-regular if for any $f \in \mathscr{C}(\partial U)$ there is a limit $\lim _{U \ni y \rightarrow x} H_{f}^{U}(y)\left[6\right.$, p. 357]. The set of semi-regular points of $U$ is denoted by $U_{\text {sem }}$. It was proved in [6, Corollary 5] that $U_{\text {sem }}$ is open in $\partial U$.

Corollary 2.6. The absorbent condition holds for $\partial \bar{U} \backslash U_{\text {sem }}$. Consequently, if $X$ is elliptic, there exist no semi-regular points on $\partial \bar{U}$.

Proof. Since $U_{\text {sem }} \cap \partial \bar{U}$ is open and $U_{\text {sem }} \subset U_{\text {ir }}$,

$$
U_{\text {sem }} \cap \partial \bar{U} \subset \partial \bar{U} \backslash U_{s} .
$$

Thus the conclusion follows from Proposition 2.5.

The converse of Corollary 2.3 does not hold: There exist nonelliptic spaces where the set $U_{s}$ is dense on $\partial \bar{U}$ for every open relatively compact $U$, as shown by the following example.

Example 2.7. Let $X^{\prime}$ be the harmonic space defined in [3, Exercise 3.2.11]. Then

$$
X:=\left\{(x, y) \in X^{\prime} \mid y \geqq 0\right\}
$$

is an absorbent set of $X^{\prime}$ and can be regarded as a harmonic space with the corresponding hyperharmonic sheaf [3, Exercise 6.1.8]. Let

$$
Y:=\{(x, y) \in X \mid y=0\} ;
$$

$Y$ is also an absorbent set of $X$. Let $U \subset X$ be open and relatively compact. Then $(\partial \vec{U} \cap Y)_{i n t}=\emptyset$ if $U$ is seen as a subset of $X$. The set $U_{s}$ must be dense on $\partial \bar{U}$, since the only nontrivial local and global absorbent sets of $X$ are contained in $Y$.

Remark 2.8. All the results of this section only apply to those boundary points of $U$ that are located on $\partial \bar{U}$. For example, if $X$ is elliptic, $U_{\text {reg }}$ is not always dense on $\partial U$, as can be seen by taking $U$ such that $\partial U \cap(\bar{U})_{\text {int }}$ contains isolated polar points. For every $U$, we even obtain $\partial U \cap(\bar{U})_{i n t} \subset \partial U \backslash U_{s}$.

By Lemma 1.7 every closed subset of $U_{i r}$ is totally thin. Those totally thin sets which are closed and contain only irregular points for some open set can be regarded as a special class of exceptional sets. The connection between the results of Sections 1 and 2 is made clear by the following corollary.

Corollary 2.9. Let $X$ be connected and $E$ a closed set of $X$ such that $X \backslash E=$ $U_{1} \cup U_{2}$, with $U_{1}, U_{2}$ open and nonempty such that $U_{1} \cap U_{2}=\emptyset$. If $E \cap \partial \bar{U}_{1}$ is a totally thin set containing only irregular points of $U_{1}$, then $\bar{U}_{1}$ is a non-trivial ab sorbent set of $X$. 
Proof. Since all irregular points are unstable, the assertion follows from Proposition 2.1.

Remark 2.10. Corollary 2.9 remains valid if $E \cap \partial \bar{U}_{1}$ contains only unstable points of $U_{1}$. In this case $E \cap \partial \bar{U}_{1}$ is not necessarily totally thin.

\title{
References
}

[1] BERG, C.: Quelques propriétés de la topologie fine dans la théorie du potentiel et des processus standard. - Bull. Sci. Math. 95, 1971, 27-31.

[2] BliedtNer, J., and W. HANSEn: Simplicial cones in potential theory. - Invent. Math. 29, 1975, 83-110.

[3] Constantinescu, C., and A. Cornea: Potential theory on harmonic spaces. - Die Grundlehren der mathematischen Wissenschaften 158. Springer-Verlag, Berlin-Heidelberg-New York, 1972.

[4] Efrros, E. G., and J. L. Kazdan: Applications of Choquet simplexes to elliptic and parabolic boundary value problems. - J. Differential Equations 8, 1970, 95-134.

[5] HeRvÉ, R.-M.: Recherches axiomatiques sur la théorie des fonctions surharmoniques et du potentiel. - Ann. Inst. Fourier (Grenoble) 12, 1962, 415-571.

[6] LUKEŠ, J., and J. MALÝ: On the boundary behaviour of the Perron generalized solution. - Math. Ann. 257, 1981, 355-366.

[7] LuKEŠ, J., and L. ZAJČEK: Connectivity properties of fine topologies. - Rev. Roumaine Math. Pures Appl. 22, 1977, 679-684.

\author{
University of Helsinki \\ Department of Mathematics \\ SF-00100 Helsinki \\ Finland
}

Received 10 April 1986 